\title{
Scale-dependent Polarization of Solar Wind Velocity Fluctuations at the Inertial and Kinetic Scales
}

\author{
Jana Šafránková ${ }^{1}$ (i), Zdeněk Němeček ${ }^{1}$, František Němec ${ }^{1}$, Daniel Verscharen ${ }^{2,3}{ }^{(10}$, Christopher H. K. Chen ${ }^{4}$, \\ Tereza Durovcová ${ }^{1}$, and Maria O. Riazantseva ${ }^{5}$ \\ ${ }^{1}$ Charles University, Faculty of Mathematics and Physics, V Holešovičkách 2, 18000 Prague 8, Czech Republic; jana.safrankova@mff.cuni.cz \\ ${ }^{2}$ Mullard Space Science Laboratory, University College London, Dorking RH5 6NT, UK \\ ${ }^{3}$ Space Science Center, University of New Hampshire, Durham, NH 03824, USA \\ ${ }^{4}$ School of Physics and Astronomy, Queen Mary University of London, London E1 4NS, UK \\ ${ }^{5}$ Space Research Institute of the Russian Academy of Sciences, Moscow, Russia, Profsoyuznaya str. 84/32, Moscow 117997, Russia \\ Received 2018 September 4; revised 2018 November 12; accepted 2018 November 17; published 2019 January 4
}

\begin{abstract}
We study the polarization properties of the velocity fluctuations in solar wind turbulence using high-resolution data from the Spektr-R spacecraft. The ratio of perpendicular to parallel velocity fluctuations in the inertial range is smaller than the equivalent ratio for magnetic fluctuations, but gradually increases throughout this range. In the kinetic range, there is a large decrease in the ratio, similar to the magnetic fluctuations. We compare the measurements to numerical solutions for a combination of kinetic Alfvén waves and slow waves, finding that both the slow increase and sharp decrease in the ratio are consistent with a majority population of Alfvén waves and minority population of slow waves in critical balance. Furthermore, the beta-dependence of this scale-dependent ratio can be successfully captured in the model when incorporating a beta-dependent Alfvén to slow wave ratio similar to that observed in the solar wind.
\end{abstract}

Key words: solar wind - turbulence - waves

\section{Introduction}

The solar wind is a turbulent plasma (Goldstein et al. 1995; Horbury et al. 2005; Alexandrova et al. 2013; Bruno \& Carbone 2013; Chen 2016) that emanates from the solar corona and carries the frozen-in magnetic field into the heliosphere. Turbulent solar wind fluctuations are characterized by a broad power spectrum that covers timescales from several hours to about $0.01 \mathrm{~s}$ (e.g., Coleman 1968). Observations gathered at different distances from the Sun also show that the solar wind expansion is non-adiabatic and the turbulent cascade is considered to be one of the important sources of energy needed to maintain this non-adiabatic behavior.

Large scale Alfvénically polarized fluctuations generated at or near the Sun propagate predominantly away from it (e.g., Belcher \& Davis 1971) into interplanetary space and interact with the sunward-propagating fluctuations generated everywhere along the solar wind path. This interaction drives a turbulent cascade (e.g., Howes 2015). This cascade transports energy from large scales where it is injected to smaller scales (e.g., Goldstein et al. 1995) until the ion gyroscale is reached. Below the ion gyroradius, a new range of turbulence carries energy to yet smaller scales where it is dissipated (Alexandrova et al. 2008; Howes et al. 2008; Sahraoui et al. 2009; Schekochihin et al. 2009; Chen et al. 2010). Nevertheless, this energy transport is not fully understood, and there are still many open questions about the nature of the fluctuations, the turbulent energy cascade, and the dissipation processes.

Zank et al. (2017) developed a model of turbulent transport from large scales to and through the inertial range based on the nearly incompressible magnetohydrodynamic (MHD) approach. This turbulence-transport model describes the collapse of the energy cascade to a highly oblique twodimensional component and a quasi-parallel slab component. Adhikari et al. (2017) enhanced this model and calculated the evolution of the anisotropy of magnetic field fluctuations with a distance from the Sun. The anisotropy increases in the transition from the energy-containing to inertial scales. Zank et al. (2018) argue that both quasi-2D and slab modes are generated at the Sun. Their interaction with the inward propagating waves contributes to solar wind heating and quasi-2D turbulence is more effective in the heating according to Zank et al. (2018).

The power spectra of magnetic field and velocity fluctuations are generally interpreted in terms of MHD turbulence theory (e.g., Goldreich \& Sridhar 1995; Galtier et al. 2000; Boldyrev 2006; Schekochihin et al. 2009) for scales larger than the ion gyroradius, where the fluctuations are predominantly Alfvénic (e.g., Belcher \& Davis 1971; Horbury et al. 1995; Bale et al. 2005). These scales are known as the inertial range, and the spectral indexes at 1 au are observed to be close to $-5 / 3$ for the magnetic field and $-3 / 2$ for velocity (e.g., Matthaeus \& Goldstein 1982; Bale et al. 2005; Podesta et al. 2007; Tessein et al. 2009; Chen et al. 2011), although they depend on other parameters such as the level of cross-helicity (Podesta \& Borovsky 2010; Chen et al. 2013). Note, however, that Roberts (2010) measured the velocity spectra from 0.3 to $5 \mathrm{au}$ and found that the spectral index becomes close to $-5 / 3$ at 5 au.

Besides the incompressible Alfvénic component that dominates turbulence in the inertial range, the solar wind also contains a small fraction (around 2\%-3\%) of energy in compressible fluctuations (e.g., Bruno \& Carbone 2013; Riazantseva et al. 2015; Chen 2016). The compressive fluctuations are slow mode polarized (Howes et al. 2012; Verscharen et al. 2017) and highly elongated along the mean-field direction (Chen et al. 2012; Chen 2016). According to predictions based on gyrokinetic theory, they are passive to Alfvénic fluctuations but have no (or little) parallel cascade along the magnetic field direction (Schekochihin et al. 2009).

An important way to investigate the nature of turbulence is to determine the polarization of the fluctuations, including the 
amplitude ratios and phase relations between the different electromagnetic fields and particle velocity moments. As discussed above, it is well known that the perpendicular magnetic fluctuations are generally much larger than the parallel magnetic fluctuations at the inertial range (e.g., Belcher \& Davis 1971; Horbury et al. 1995). In the kinetic range, the magnetic compressibility increases, consistent with a transition to kinetic Alfvén turbulence (Salem et al. 2012; TenBarge et al. 2012; Kiyani et al. 2013). Recently, it has been shown that the magnetic compressibility increases again at the electron inertial scale when the electron temperature is much less than the proton temperature, consistent with a transition to inertial kinetic Alfvén turbulence (Chen \& Boldyrev 2017).

The polarization of the velocity fluctuations has been less comprehensively studied. In particular, the ratio of perpendicular to parallel fluctuations in the kinetic range has not previously been studied due to the lack of high-resolution velocity data. This paper presents the first measurement of this ratio and its dependence on the plasma $\beta$ using data from the Spektr- $R$ spacecraft. A survey of about 35,000 subintervals indicates that the velocity fluctuations are more compressive at smaller scales, in agreement with theoretical predictions for the kinetic wave modes. Moreover, we have found that the compressibility of the fluctuations increases with proton $\beta$ and that they become isotropic when proton $\beta$ approaches unity.

\section{Observational Data}

The analysis concentrates on ion velocity fluctuations at frequencies covering the transition from the inertial range to the kinetic range. The velocity components are determined from measurements made by the six Faraday cups (FCs) of the Bright Monitor of Solar Wind instrument onboard the Spektr-R spacecraft (Šafránková et al. 2013). Three FCs are oriented away from the instrument axis and serve for a determination of the total ion flux vector. The other three point toward the Sun and are equipped with deceleration grids to provide three points of the ion distribution with a $32 \mathrm{~Hz}$ cadence. They are used for a determination of the proton speed and temperature in the adaptive mode. With these data, the time resolution is sufficient for a spectral analysis up to $16 \mathrm{~Hz}$ but the present paper is limited to $2 \mathrm{~Hz}$ in order to guarantee that the power spectral densities (PSDs) are well above a noise level, even in intervals of low-velocity variations (Šafránková et al. 2016).

The analyzed data set covers the years 2011-2016 but the intervals used are determined by the availability of Spektr-R measurements in the adaptive mode, which occurs about $10 \%-15 \%$ of the observation time. The analysis is performed on the solar wind measurements that are divided into 20 minutes basic subintervals with the overlapping duration 19 minutes and the fast Fourier transform is computed on each subinterval (Šafránková et al. 2015). The Spektr-R magnetometer is not in operation, thus we use hightime resolution magnetic field data from the Wind spacecraft (Lepping et al. 1995). The time of Spektr-R observations is propagated upstream to the location of Wind in order to estimate the corresponding intervals in Wind magnetic field measurements. Since Wind and Spektr-R are not necessarily in the same solar wind stream, we analyze only the intervals when the difference between the solar wind speeds determined by Wind and Spektr-R is lower than 5\%. These speeds lie between the two dashed lines in Figure 1 (left), which shows a comparison of the solar-wind speeds from Wind and Spektr-R.
This procedure leaves 35,937 intervals for further processing. Figure 1 (right panel) shows the distribution of angles $\theta$ between the GSE $x$-axis and the velocity measured using both Wind and Spektr-R. The distribution is similar and suggests that the magnetic field can be propagated between the two spacecraft locations, and possibly be used as the mean field direction when defining the parallel and perpendicular velocity components.

\section{Results}

The PSDs of the parallel and perpendicular components of both the magnetic field, $B$ and velocity, $V$ are calculated for all intervals in our data set. A 20 min magnetic field average is used as the mean field direction and this direction is also used for the separation of parallel and perpendicular velocity components in corresponding intervals of the Spektr-R measurements. The median spectra are shown in Figure 2 and their ratios in Figure 3. The magnetic field results are shown in Alfvén units, $B^{\text {normalized }}=B /\left(\mu_{0} \cdot \rho\right)^{1 / 2}$ and all frequencies are normalized to the scale

$$
f_{d}=\frac{V_{\mathrm{sw}}}{2 \pi\left(\rho_{p}+d_{p}\right)}
$$

before the median values are calculated. In the equation, $f_{d}$ is the normalized frequency, $V_{\mathrm{sw}}$ is the solar wind bulk speed, $\rho_{p}$ is the proton thermal gyroradius $\left(\rho_{p}=V_{\mathrm{th}} / \omega_{c}\right.$ where $V_{\mathrm{th}}$ is the thermal velocity, and $\omega_{c}$ is proton cyclotron frequency), and $d_{p}$ is the proton inertial length. This scale contains a mixture of the proton gyroradius, $\rho_{p}$ and inertial length and has been found empirically to describe the location of the ion break scale over a wide range of $\beta$ values (e.g., Šafránková et al. 2016; Woodham et al. 2018). Leamon et al. (1998) argue that this scale corresponds to the wavenumber at which the cyclotron dissipation becomes effective.

In Figures 2 and 3, the energy in fluctuations of $B_{\perp}$ compared to that in fluctuations of $B_{\|}$is greater by a constant factor of about 15 within the inertial range but the ratio decreases to about 3 through the kinetic range. This is consistent with previous findings for the magnetic field fluctuations (e.g., Salem et al. 2012; TenBarge et al. 2012; Kiyani et al. 2013). The right-hand panel shows a similar ratio for the velocity fluctuations using both Wind (red curve) and Spektr-R (black curve). The good agreement of the PSDs in the range of overlap shows that the Wind mean magnetic field direction can be used at the locations of both spacecraft and any evolution of the compression ratio from L1 to the Earth is negligible. A key new finding here is that, similarly to the magnetic field, the velocity compression ratio also sharply decreases as the kinetic range is reached (between 0.4 and $2 \mathrm{~Hz}$ ). However, unlike the magnetic field, there is a steady increase in the ratio $V_{\perp}^{2} / V_{\|}^{2}$ (where $V_{\perp}$ and $V_{\|}$are the amplitudes of velocity fluctuations perpendicular and parallel to the background magnetic field, $B_{0}$, respectively) throughout the MHD inertial range. A model for, and interpretation of, these two new features is described in Section 4.

It is also of interest to determine the spectral indices of the PSDs shown in Figure 2, which are given in Table 1. The slopes and the break are determined in one step as the best fit with two straight lines to the median spectra in Figure 2 and the break scale corresponds to their intersection. Note that all spectra are computed in the frequency range of $10^{-3}$ to $2 \mathrm{~Hz}$ 

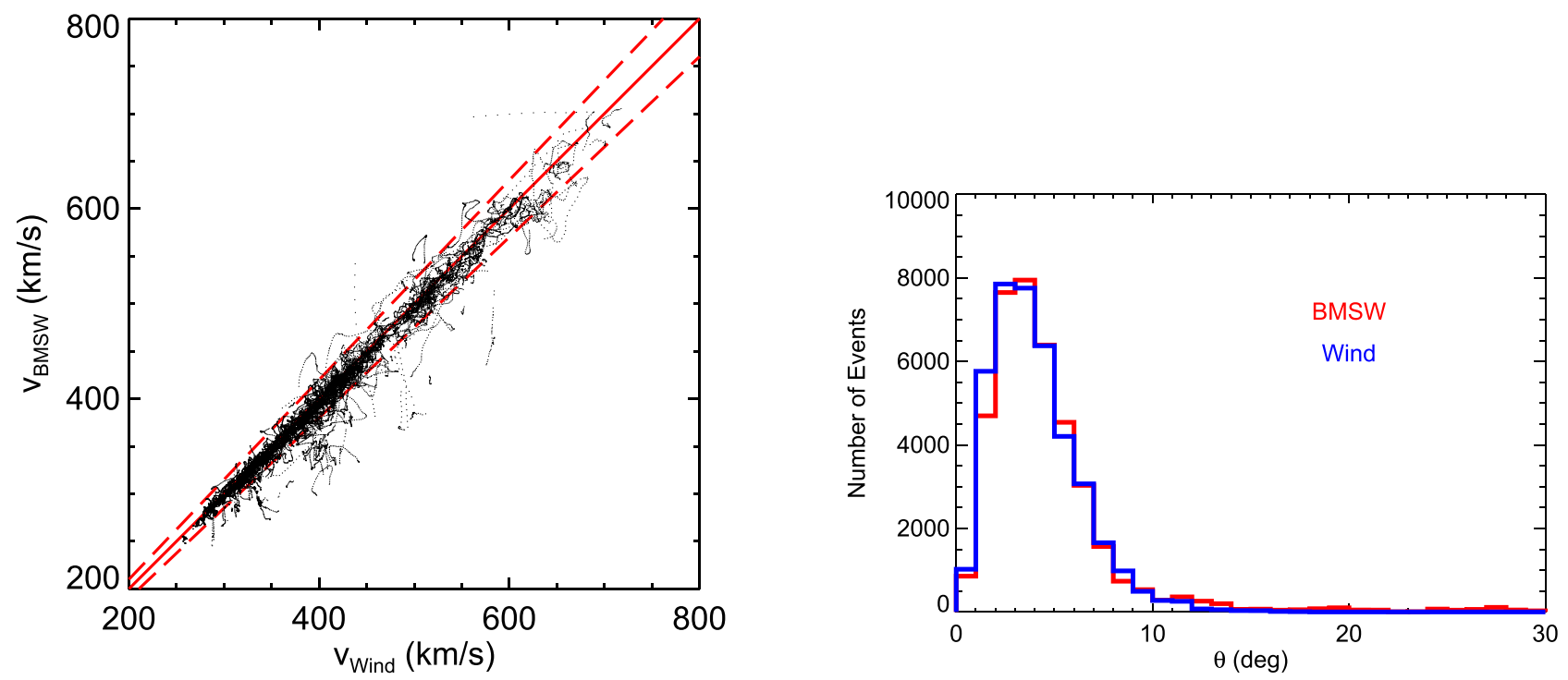

Figure 1. Comparison of Wind and Spektr-R proton speed measurements (left) and histograms of the angle $\theta$ between the solar wind velocity and the GSE $x$-axis (right) in both locations.
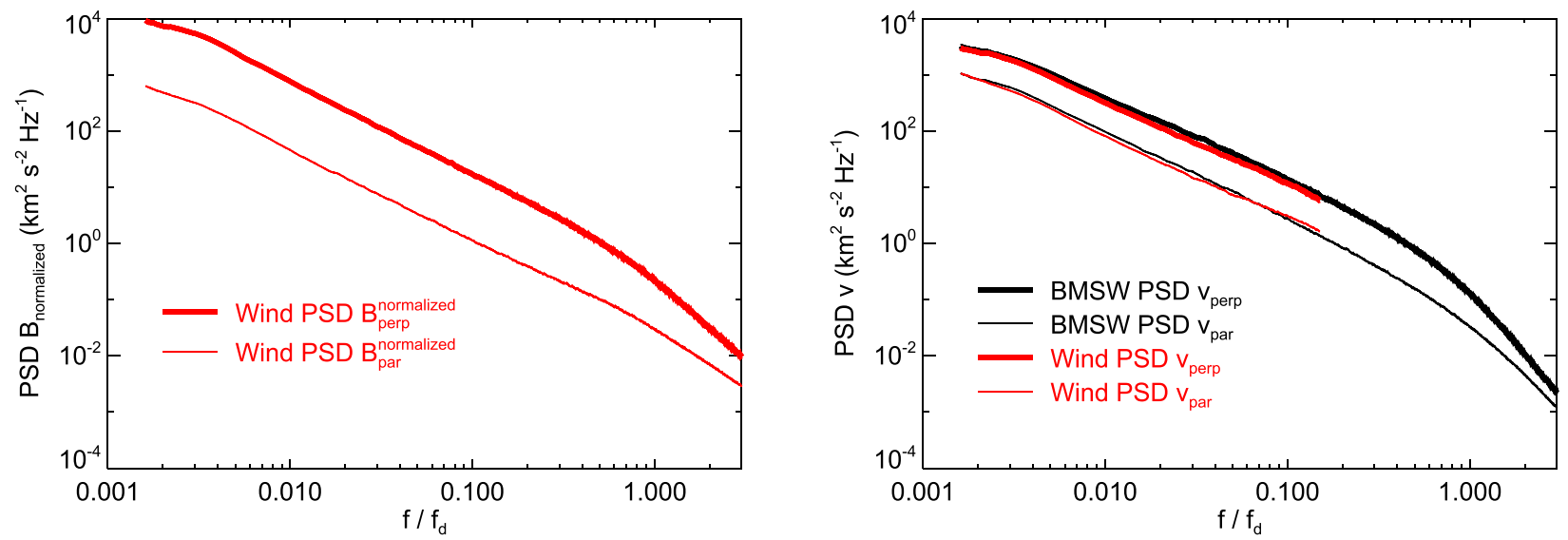

Figure 2. Median frequency spectra of both $B_{\perp}$ (heavy line) and $B_{\|}$(thin line) magnetic field components (left) and $V_{\perp}$ (heavy line) and $V_{\|}$(thin line) velocity (right) fluctuations. In the right-hand panel, the black and red colors correspond to Spektr-R and Wind velocities, respectively.
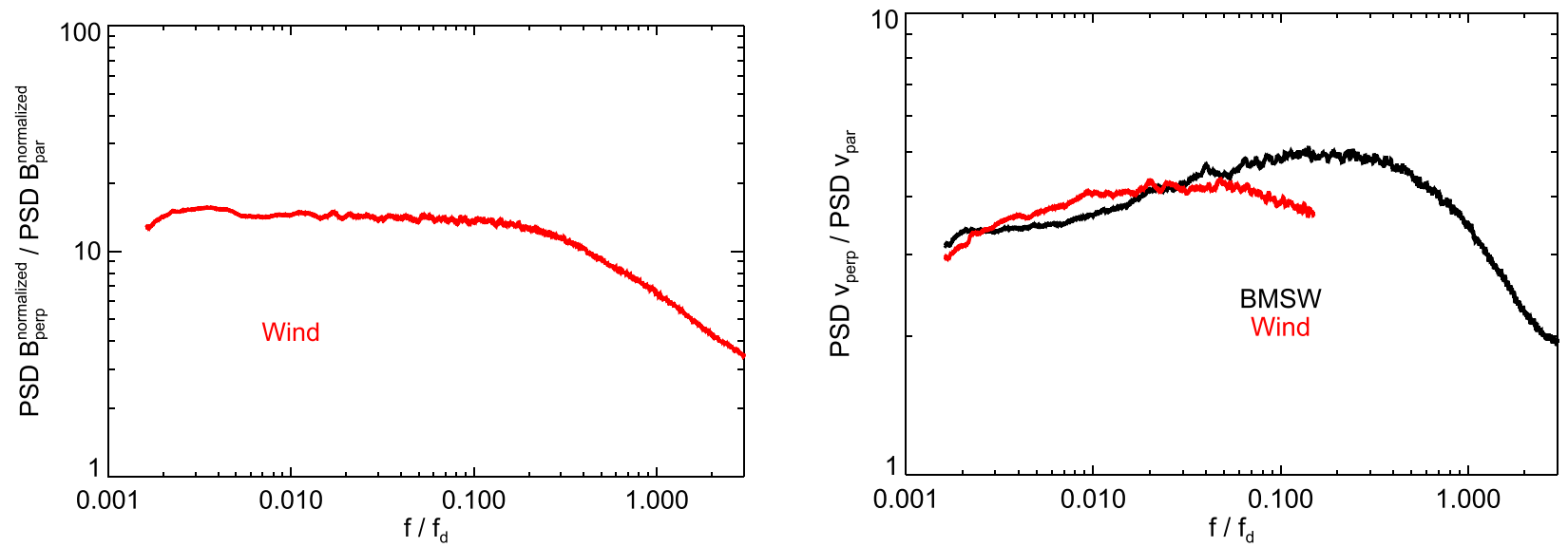

Figure 3. Ratio of PSDs of the $B_{\perp}$ and $B_{\|}$magnetic field fluctuations (left) and the same plot for $V_{\perp}$ and $V_{\|}$velocity fluctuations (right). In the right-hand panel, the black and red colors correspond to Spektr-R and Wind velocities, respectively.

but the normalization to $f_{d}$ shifts the frequency axes. Since the number of spectra from which the median is computed is rather low at the lowest and highest $f / f_{d}$, we limit the range of fitting to $0.03<f / f_{d}<3$.
In the inertial range, both $B_{\perp}$ and $B_{\|}$have spectral indices close to $-5 / 3$, and $V_{\perp}$ and $V_{\|}$have spectral indices close to $-3 / 2$. These values are consistent with previous measurements of the solar wind turbulent cascade. In the kinetic range, the 
Table 1

Parameters of Fits to the Median Frequency Spectra from Figure 2

\begin{tabular}{lcrrr}
\hline \hline & $B_{\perp}$ & \multicolumn{1}{c}{$B_{\|}$} & \multicolumn{1}{c}{$V_{\perp}$} & \multicolumn{1}{c}{$V_{\|}$} \\
\hline Inertial range spectral index & -1.63 & -1.57 & -1.45 & -1.55 \\
Kinetic range spectral index & -2.68 & -2.11 & -3.25 & -2.66 \\
Break scale & 0.53 & 0.89 & 0.44 & 0.44 \\
\hline
\end{tabular}

slope of $B_{\perp}$ is close to $-8 / 3$, and $V_{\perp}$ is -3.2 , again consistent with previous findings. The parallel components of both fields have shallower slopes than the perpendicular components in the kinetic range, although this may be because the fitting range is close to the transition region and not representative of the asymptotic spectra. The normalized break scales, determined as the intersection of the two power-law fits, are close to $0.5 f_{d}$ for all analyzed parameters except $B_{\|}$. However, the difference between the slopes of the two parts of the $B_{\|}$spectrum is rather small and thus even a very small uncertainty in the slope determination results in a large error in the break frequency. For this reason, we consider a break scale of $\approx 0.5 f_{d}$ to be appropriate for all spectra.

Finally, we investigate the beta dependence of the results. Each of the 20 min intervals corresponds to different magnetic field and plasma conditions (mean magnetic field, temperature, density, etc.), which change the characteristics of turbulence. The majority of the intervals correspond to a relatively narrow range of proton $\beta$ centered around 0.3 ; however, we divide them into six bins, with an approximately equal number of intervals in each, and compute the median PSD ratios. The results are shown in Figure 4.

The figure shows that the PSD $B_{\perp} / B_{\|}$ratio decreases as proton $\beta$ approaches unity. A similar result holds for the velocity fluctuations, with the positive slope in the inertial range being more prominent at low $\beta$. To understand the origin of this behavior, we construct a model for the turbulent field ratios in the following section.

\section{Numerical Model}

We calculate the polarization relations for velocity fluctuations from a combination of Alfvén waves and slow waves using the hot-plasma dispersion relation based on the linearized Vlasov-Maxwell equations with the NHDS code (Verscharen \& Chandran 2013, 2018; Verscharen et al. 2016, 2017). We assume that the turbulent fluctuations inherit these linear polarization relations, even though we do not require that turbulence consist of linear waves.

We set the turbulent spectrum of $B_{\perp}$ as

$$
\operatorname{PSD} \quad B_{\perp} \propto \begin{cases}k_{\perp}^{-5 / 3}, & \text { if } \quad k_{\perp}<1 / \rho_{p}, \\ k_{\perp}^{-7 / 3}, & \text { if } \quad k_{\perp} \geqslant 1 / \rho_{p}\end{cases}
$$

where $\rho_{p}$ is the proton gyroradius and $k_{\perp}$ is the wavevector component perpendicular to the background magnetic field, $B_{0}$. We note that the observed spectral break occurs at $f / f_{d} \approx 0.5$ (Table 1), which corresponds to a break wavenumber of order $1 / \rho_{p}$ when $\beta \approx 1$.

Although our results are independent of the absolute amplitude of PSD $B_{\perp}$, we choose it so that the spectrum is continuous at $k_{\perp}=1 / \rho_{p}$. We then define the large-scale magnetic compressibility as the ratio, $r=B_{\|, 0} / B_{\perp, 0}$, where $B_{\|, 0}\left(B_{\perp, 0}\right)$ is the amplitude of parallel (perpendicular) magnetic field fluctuations at a scale much greater than $1 / \rho_{p}$. We assume that $r$ is constant in the inertial range.

We relate $k_{\|}$to $k_{\perp}$ according to the critical-balance condition (Goldreich \& Sridhar 1995) as

$$
k_{\|} \propto \begin{cases}k_{\perp}^{2 / 3}, & \text { if } \quad k_{\perp}<1 / \rho_{p}, \\ k_{\perp}^{1 / 3}, & \text { if } \quad k_{\perp} \geqslant 1 / \rho_{p}\end{cases}
$$

and fix the proportionality constants in Equation (3) so that $k_{\|}$ is continuous at $k_{\perp} \rho_{p}=1$, and $k_{\|}=k_{\perp}$ at the outer scale (assumed as $k_{\perp}=5 \times 10^{-4} / d_{p}$, where $d_{p}$ is the proton inertial length). This leads to $\theta$ varying between $59^{\circ}$ and $88^{\circ}$, where $\theta$ is the angle between $\boldsymbol{k}$ and $\boldsymbol{B}_{0}$. For the NHDS calculations, we set $V_{A} / c=10^{-4}$ and assume that both protons and electrons are isotropic and have equal temperatures. We then calculate the ratios between velocity fluctuations and magnetic field fluctuations using linear theory depending on $k_{\perp}$ and $k_{\|}$.

We connect the magnetic field spectrum with the velocity spectrum through the squares of the amplitude ratios for Alfvén waves (index $A$ ) and slow waves $(S)$ where the indices $A$ and $S$ refer to the full $\boldsymbol{k}$-dependent amplitude ratios as

$$
\operatorname{PSD} V_{\perp}=\left[\left(\left.\frac{V_{\perp}}{B_{\perp}}\right|_{A}\right)^{2}+\left(\left.r \frac{V_{\perp}}{B_{\|}}\right|_{S}\right)^{2}\right] \operatorname{PSD} B_{\perp}
$$

and

$$
\operatorname{PSD} V_{\|}=\left[\left(\left.\frac{V_{\|}}{B_{\perp}}\right|_{A}\right)^{2}+\left(\left.r \frac{V_{\|}}{B_{\|}}\right|_{S}\right)^{2}\right] \operatorname{PSD} B_{\perp} .
$$

For each $\beta$, we set $r$ to the value that generates the best agreement between our results in the low-frequency limit and the observations from Figure 4. We show the results of our calculation for PSD $V_{\perp} / \mathrm{PSD} V_{\|}$as functions of $f / f_{d}$ in Figure 5.

By comparing Figures 4 and 5, we find that our model describes the observed frequency dependence of PSD $V_{\perp} / \mathrm{PSD}$ $V_{\|}$to a reasonable degree. The large-scale magnetic compressibility $r$ and the characteristic size of the outer scale determine the value of PSD $V_{\perp} / \mathrm{PSD} V_{\|}$at low frequencies. The frequency-dependent anisotropy according to Equation (3) then determines the frequency dependence of PSD $V_{\perp} / \mathrm{PSD} V_{\|}$ until $f \approx f_{d}$. We also checked a fixed anisotropy but it leads to a roughly constant PSD $V_{\perp} / \mathrm{PSD} V_{\|}$in the inertial range. In the kinetic range, PSD $V_{\perp} / \mathrm{PSD} V_{\|}$decreases due to the increasing compressibility of the Alfvén wave at kinetic scales (kinetic Alfvén wave).

From Figure 4 (left), we achieve an estimate for the value of $r$ as the low-frequency limit of $\tilde{r} \sim 1 / \sqrt{\left(\operatorname{PSD} B_{\perp} / \operatorname{PSD} B_{\|}\right)}$. These values are, however, significantly greater than those leading to the best agreement in Figure 5. We assume that this difference is a consequence of the overestimation of PSD $B_{\|}$in the measurements due to inaccuracies in the determination of the parallel direction when the fluctuation level is comparable with the $B_{0}$ background magnetic field as in the solar wind. To overcome this problem, the ratio PSD $B_{\perp} / \mathrm{PSD}|B|$ was used as a measure of compressibility. We found that the compression factor determined in this way is by a factor of about 1.6 lower than that computed from $B_{\perp}$ and $B_{\|}$but still larger by a factor of $\approx 2$ than that used in the model. Nevertheless, the overall increasing trend of the compression ratio, $r$ on $\beta$, used in the 

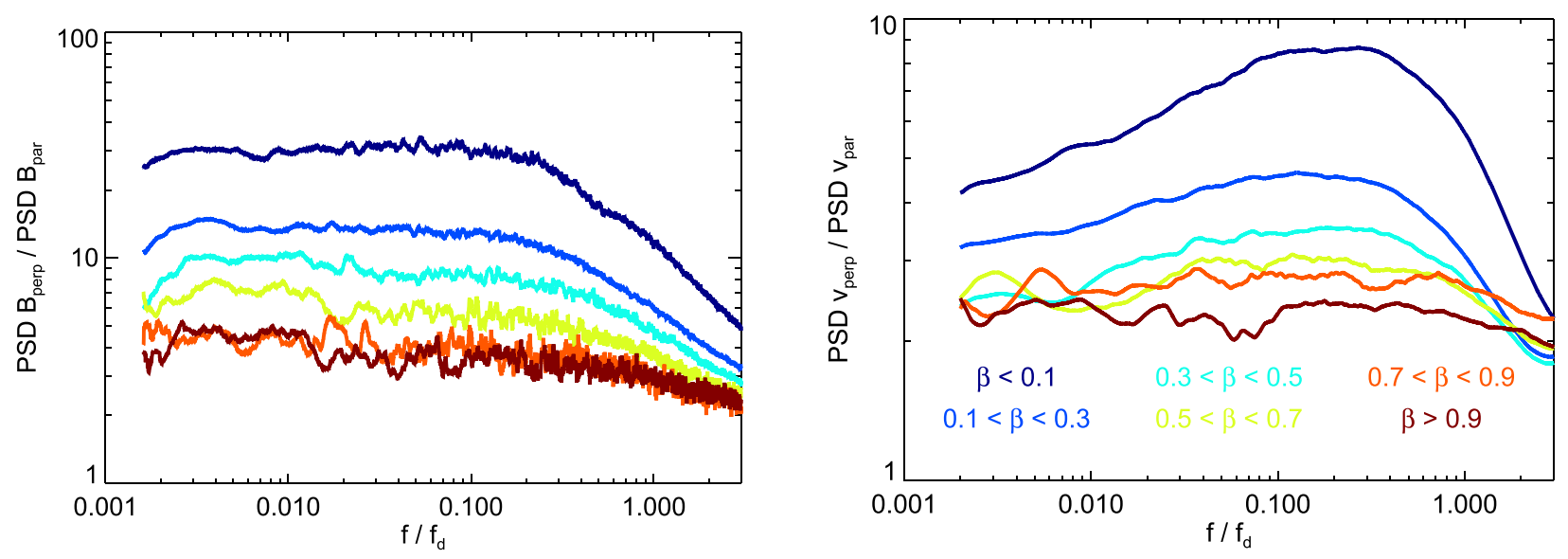

Figure 4. Ratio of PSDs $B_{\perp} / B_{\|}$(left) and PSDs $V_{\perp} / V_{\|}$(right) as a function of $f / f_{d}$. The colors mark different ranges of the proton $\beta$; the scale is explained in the righthand panel.

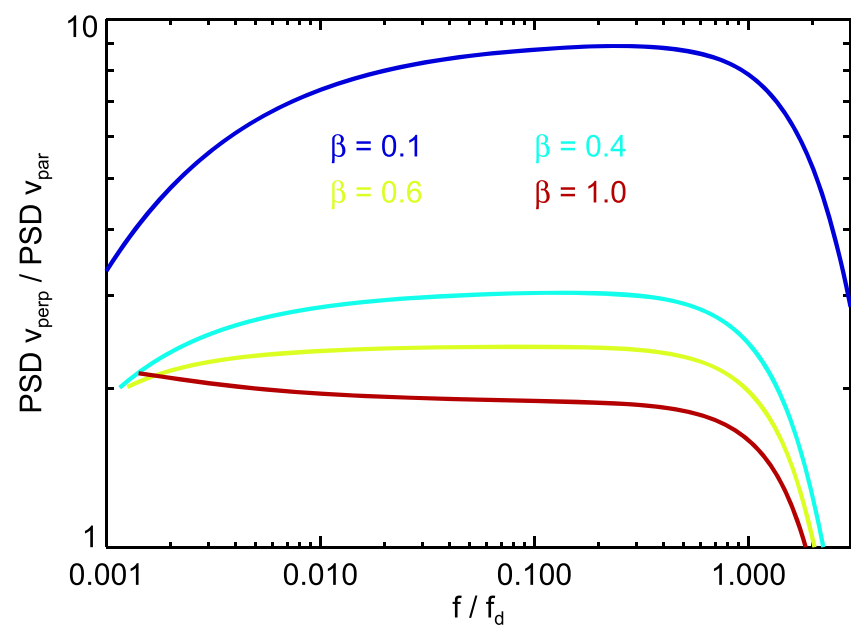

Figure 5. Calculated ratios of PSDs of the $V_{\perp}$ and $V_{\|}$velocity fluctuations. We apply a $\beta$-dependent large-scale magnetic compressibility, $r(r=0.055$ at $\beta=0.1, r=0.12$ at $\beta=0.4, r=0.13$ at $\beta=0.6$, and $r=0.13$ at $\beta=1$ ).

model is consistent with observations; the reason for the quantitative difference could be nonlinear effects.

\section{Discussion and Conclusion}

The FCs onboard Spektr- $R$ allow us to observe velocity fluctuations up to a frequency of $2 \mathrm{~Hz}$. For the first time, these data provide the opportunity to study the ratio of perpendicular to parallel velocity fluctuations in solar-wind turbulence on small scales. Our comparison between the observed ratio PSD $V_{\perp} / \mathrm{PSD} V_{\|}$and the results of the linear theory based on critical balance and a superposition of Alfvén waves and slow waves shows a good agreement. The results are consistent with a composition of Alfvénic/kinetic-Alfvénic and slow-modelike turbulence over a wide range of scales. This finding corroborates previous theoretical and observational works on the nature of solar-wind turbulence.

Since the Spektr-R magnetometer is not operational, we combine magnetic field data from the Wind spacecraft and fluctuations in the plasma velocity from the Spektr-R spacecraft to study the polarization properties of inertial-range and protonscale turbulence in the solar wind. We show that it is possible to propagate the information on the interplanetary magnetic field (IMF) direction from L1 to Earth if the observing spacecraft sample the same wind stream. In agreement with previous studies, we find that the slopes of the median magnetic field spectra in the inertial ranges are close to $-5 / 3$ for both the perpendicular and the parallel components of the fluctuations. The slopes do not depend on proton $\beta$. The break between scales occurs at $k \approx 1 /\left(\rho_{p}+d_{p}\right)$ for all analyzed components. The slope of the median spectrum of the perpendicular magnetic field fluctuations is approximately $-8 / 3$ below the break point, while the spectrum of the parallel magnetic field fluctuations is flatter with a power-index less than $-7 / 3$.

In linear theory, slow waves undergo strong Landau damping which is probably responsible for steeper slopes of the modeled velocity spectra than those observed in the kinetic range. Nevertheless, the basic trend of the evolution of the slope with increasing $\beta$ is the same as the trend in the observations. There is some evidence, however, that fluctuations in the solar wind behave more fluid-like than expected for a weakly collisional plasma (Verscharen et al. 2017; Wu et al. 2018). This effect can lead to a suppression of Landau damping and explain some of the deviations between our observational and model results. A comparison of our kinetic results with large-scale and small-scale fluid theory may resolve these deviations and the beta-dependence of $r$.

The authors thank the Wind team for the magnetic field data. The BMSW data are available via http://aurora.troja.mff.cuni. $\mathrm{cz} /$ spektr-r/project/. The work of the Czech authors was supported by the Czech Science Foundation under Contract 1604956S. C.H.K.C. is supported by STFC Ernest Rutherford Fellowship ST/N003748/2, and D.V. is supported by STFC Ernest Rutherford Fellowship ST/P005489/1.

Facilities: Spektr-R.

\section{ORCID iDs}

Jana Šafránková (i) https://orcid.org/0000-0003-4178-5206 Daniel Verscharen (1) https://orcid.org/0000-0002-0497-1096

\section{References}

Adhikari, L., Zank, G. P., Telloni, D., et al. 2017, ApJ, 851, 117

Alexandrova, O., Carbone, V., Veltri, P., \& Sorriso-Valvo, L. 2008, ApJ, 74,1153

Alexandrova, O., Chen, C. H. K., Sorriso-Valvo, L., Horbury, T. S., \& Bale, S. D. 2013, SSRv, 178, 101 
Bale, S. D., Kellogg, P. J., Mozer, F. S., Horbury, T. S., \& Reme, H. 2005, PhRvL, 94, 215002

Belcher, J. W., \& Davis, L. 1971, JGR, 76, 3534

Boldyrev, S. 2006, PhRvL, 96, 115002

Bruno, R., \& Carbone, V. 2013, LRSP, 10, 2

Chen, C. H. K. 2016, JPIPh, 82, 535820602

Chen, C. H. K., Bale, S. D., Salem, C. S., \& Maruca, B. A. 2013, ApJ, 770, 125

Chen, C. H. K., Bale, S. D., Salem, C. S., \& Mozer, F. S. 2011, ApJL, 737, L41

Chen, C. H. K., \& Boldyrev, S. 2017, ApJ, 840, 122

Chen, C. H. K., Horbury, T. S., Schekochihin, A. A., et al. 2010, PhRvL, 104 255002

Chen, C. H. K., Mallet, A., Schekochihin, A. A., et al. 2012, ApJ, 758, 120

Coleman, P. J. 1968, ApJ, 153, 371

Galtier, S., Nazarenko, S. V., Newell, A. C., \& Pouquet, A. 2000, JPIPh, 63, 447

Goldreich, P., \& Sridhar, S. 1995, ApJ, 438, 763

Goldstein, M. L., Roberts, D. A., \& Matthaeus, W. H. 1995, ARA\&A, 33, 283

Horbury, T. S., Balogh, A., Forsyth, R. J., \& Smith, E. J. 1995, GeoRL, 22,3401

Horbury, T. S., Forman, M. A., \& Oughton, S. 2005, PPCF, 47, B703

Howes, G. G. 2015, RSPTA, 373, 2041

Howes, G. G., Bale, S. D., Klein, K. G., et al. 2012, ApJL, 753, L19

Howes, G. G., Cowley, S. C., Dorland, W., et al. 2008, JGRA, 113, A05103

Kiyani, K. H., Chapman, S. C., Sahraoui, F., et al. 2013, ApJ, 763, 10

Leamon, R. J., Smith, C. W., Ness, N. F., Matthaeus, W. H., \& Wong, H. K. 1998, JGR, 103, 4775

Lepping, R. P., Acũna, M. H., Burlaga, L. F., et al. 1995, SSRv, 71, 207
Matthaeus, W. H., \& Goldstein, M. L. 1982, JGR, 87, 6011

Podesta, J. J., \& Borovsky, J. E. 2010, PhPl, 17, 112905

Podesta, J. J., Roberts, D. A., \& Goldstein, M. L. 2007, ApJ, 664, 543

Riazantseva, M. O., Budaev, V. P., Zelenyi, L. M., et al. 2015, RSPTA, 373 20140146

Roberts, D. A. 2010, JGR, 115, A12101

Šafránková, J., Němeček, Z., Němec, F., et al. 2015, ApJ, 803, 107

Šafránková, J., Němeček, Z., Němec, F., et al. 2016, ApJ, 825, 121

Šafránková, J., Němeček, Z., Přech, L., et al. 2013, SSRv, 175, 165

Sahraoui, F., Goldstein, M. L., Robert, P., \& Khotyaintsev, Y. V. 2009, PhRvL, 102, 231102

Salem, C. S., Howes, G. G., Sundkvist, D., et al. 2012, ApJL, 745, L9

Schekochihin, A. A., Cowley, S. C., Dorland, W., et al. 2009, ApJS, 182 310

TenBarge, J. M., Podesta, J. J., Klein, K. G., \& Howes, G. G. 2012, ApJ, 753,371

Tessein, J. A., Smith, C. W., MacBride, B. T., et al. 2009, ApJ, 692, 684

Verscharen, D., \& Chandran, B. D. G. 2013, ApJ, 764, 88

Verscharen, D., \& Chandran, B. D. G. 2018, RNAAS, 2, 13

Verscharen, D., Chandran, B. D. G., Klein, K. G., \& Quataert, E. 2016, ApJ, 831,128

Verscharen, D., Chen, C. H. K., \& Wicks, R. T. 2017, ApJ, 840, 106

Woodham, L. D., Wicks, R. T., Verscharen, D., \& Owen, C. J. 2018, ApJ, 856,49

Wu, H., Verscharen, D., Wicks, R. T., et al. 2018, arXiv:1808.09763

Zank, G. P., Adhikari, L., Hunana, P., et al. 2017, ApJ, 835, 147

Zank, G. P., Adhikari, L., Hunana, P., et al. 2018, ApJ, 854, 32 